\title{
CURB-65 and other markers of illness severity in community-acquired pneumonia among HIV-positive patients
}

International Journal of STD \& AIDS 2016, Vol. 27(II) 998-1004 (C) The Author(s) 2015

Reprints and permissions: sagepub.co.uk/journalsPermissions.nav DOI: 10.1 I77/09564624I5605232 std.sagepub.com

SSAGE

\author{
André Almeida ${ }^{1,2}$, Ana Rita Almeida ${ }^{3}$, Sara Castelo Branco ${ }^{1,2}$, \\ Zsófia Vesza $^{1,2}$ and Rui Pereira ${ }^{4}$
}

\begin{abstract}
As the relative burden of community-acquired bacterial pneumonia among HIV-positive patients increases, adequate prediction of case severity on presentation is crucial. We sought to determine what characteristics measurable on presentation are predictive of worse outcomes. We studied all admissions for community-acquired bacterial pneumonia over one year at a tertiary centre. Patient demographics, comorbidities, HIV-specific markers and CURB-65 scores on Emergency Department presentation were reviewed. Outcomes of interest included mortality, bacteraemia, intensive care unit admission and orotracheal intubation. A total of 396 patients were included: 49 HIV-positive and 347 HIVnegative. Mean CURB-65 score was 1.3 for HIV-positive and 2.2 for HIV-negative patients $(p<0.000 \mathrm{I})$, its predictive value for mortality being maintained in both groups ( $p=0.03$ and $p<0.001$, respectively). Adjusting for CURB-65 scores, HIV infection by itself was only associated with bacteraemia (adjusted odds ratio [AOR] 7.I, 95\% Cl [2.6-19.5]). Patients with $<200 \mathrm{CD} 4$ cells/ $\mu \mathrm{L}$ presented similar CURB-65 adjusted mortality (aOR I.7, 95\% Cl [0.2-15.2]), but higher risk of intensive care unit admission (aOR 5.7, 95\% Cl [I.5-22.0]) and orotracheal intubation (aOR 9.1, 95\% Cl [2.2-37.I]), compared to HIV-negative patients. These two associations were not observed in the $>200 \mathrm{CD} 4$ cells/ $\mu \mathrm{L}$ subgroup (aOR 2.2, 95\% Cl [0.7-7.6] and aOR 0.8, 95\% Cl [0.I-6.5], respectively). Antiretroviral therapy and viral load suppression were not associated with different outcomes $(p>0.05)$. High CURB-65 scores and CD4 counts $<200$ cells $/ \mu \mathrm{L}$ were both associated with worse outcomes. Severity assessment scales and CD4 counts may both be helpful in predicting severity in HIV-positive patients presenting with community-acquired bacterial pneumonia.
\end{abstract}

\section{Keywords}

HIV, AIDS, opportunistic infection, pneumonia, community-acquired pneumonia, clinical prediction rule, CURB-65 score

Date received: 26 May 2015; accepted: 18 August 2015

\section{Introduction}

Early since the introduction of highly active antiretroviral therapy (HAART) in the mid-1990s, morbidity and mortality among human immunodeficiency virus (HIV)-infected patients have decreased dramatically. Rates of opportunistic infections and AIDSdefining neoplasms registered a sharp decline, with a resulting drop in the number of related hospitalisations and deaths. ${ }^{1,2}$

Community-acquired pneumonia (CAP) is defined as an acute lower respiratory tract infection associated with new radiographic shadowing for which there is no other explanation and which is deemed to be community-acquired, as opposed to hospital-acquired or health care associated. ${ }^{3,4}$ HIV infection has been consistently described as a major risk factor for $\mathrm{CAP},{ }^{5-8}$ which is considered a stage 3 disease in the

\footnotetext{
'Central Lisbon Hospital Centre, Department of Internal Medicine 4, Hospital de Santa Marta, Lisbon, Portugal

${ }^{2}$ NOVA Medical School, Universidade Nova de Lisboa, Lisbon, Portugal ${ }^{3}$ Higher Institute of Applied Psychology (ISPA), Psychology and Health Research Unit, Lisbon, Portugal

${ }^{4}$ Central Lisbon Hospital Centre, Intensive Care Unit 7, Hospital Curry Cabral, Lisbon, Portugal
}

\section{Corresponding author:}

André Almeida, Serviço de Medicina Interna 4, Hospital de Santa Marta, Rua de Santa Marta, I169-I24 Lisboa, Portugal.

Email: andre.almeida@chlc.min-saude.pt 
World Health Organization HIV staging classification ${ }^{9}$ and an AIDS-defining illness by the American Centers for Disease Control and Prevention (CDC) when recurrent. ${ }^{10}$ Despite a reported decline in incidence over the last two decades, ${ }^{7-8,11}$ this condition remains frequent in this population, with its relative burden presently greater than before. ${ }^{11-14}$ In fact, CAP among HIVinfected patients has been found in some studies performed in high-income settings to be the most prevalent severe non-AIDS-defining infection and one of the most frequent reasons for hospital admission. ${ }^{11,12,15,16}$ Low CD4 cell counts, uncontrolled viral replication and absence of HAART have been associated with increased incidence. ${ }^{6,12,14-16}$

Research has addressed this topic since the beginning of the HAART era, focusing on the course and outcome of CAP in HIV-positive patients. ${ }^{5,13,15,17-19}$ HIV infection is a known risk factor for bacteraemia, ${ }^{5,19}$ but apparently not for higher mortality $y^{5,18,20,21}$ or time to clinical stability. ${ }^{20,21}$ Evidence on prediction of severity and resulting guidance on risk stratification and related management remains heterogeneous. ${ }^{13,15,17,18}$

In order to assess CAP severity on presentation and consequently guide therapeutic options, several prediction scales based on clinical and laboratorial criteria have been developed, which are nowadays widely used and endorsed by clinical societies worldwide. ${ }^{3,4,22}$ The Pneumonia Severity Index (PSI), developed in the USA, uses a total of 20 variables, including clinical background and signs, and venous and arterial blood markers. ${ }^{23}$ CURB-65, introduced by researchers from the UK, the Netherlands and New Zealand, uses a more elementary prediction rule based on four clinical and one laboratory parameter. ${ }^{3}$ Clinicians in the Emergency Department (ED) are often faced with a great deal of uncertainty in applying general clinical prediction rules to HIV-positive patients and consequently deciding site-of-care. A few observational works on this field, which evaluated the applicability of PSI in this population, have consistently found that a high score correlates with a higher risk of mortality. ${ }^{13,17,18}$ In one study designed to analyse the association between nasopharyngeal Pneumococcus density in South African HIV-positive patients with markers of disease severity and poor outcome, no correlation between CURB-65 scores and mortality was found. ${ }^{24}$ Furthermore, it has not been clearly established whether HIV infection per se should be regarded as an additional independent prognostic marker.

In this work, we aimed to assess the prognostic importance of CURB-65 in the prediction of morbidity and mortality among HIV patients presenting with CAP. Our further goal was to determine if HIV seropositivity, greater immunosuppression, HAART use and lack of virological control act as additional independent prediction factors for worse outcomes.

\section{Methods}

\section{Study design and setting}

Using a retrospective cohort design, we studied all admissions occurring over the year 2010 in a tertiary teaching hospital centre in Lisbon, Portugal, which were classified by attending ED physicians as CAP.

\section{Participants, measurements and outcomes}

Hospital electronic diagnosis code registry, clinical notes, laboratory results and chest images were reviewed. HAART prescription, lymphocyte subset and viral load data were also assessed in Si.vida, a Portuguese national network platform where updated HIV-positive patients' data are stored.

All admitted adult patients (18 years or older) who met clinical and radiological criteria for pneumonia were primarily included. Exclusion criteria comprised respiratory infections which were in the course of admission found to be caused by mycobacteria or Pneumocystis, either by molecular, microbiological or histopathological methods. Patients hospitalised within 90 days prior to admission or meeting risk factors for health care-associated pneumonia ${ }^{4}$ and patients with immunosuppression due to causes other than HIV infection (ongoing chemo- or radiotherapy, haematological malignancy, steroid use and asplenia) were also excluded.

Baseline variables included demographic data, comorbidities, HIV serostatus, lymphocyte subset count, CURB-65 score (confusion, urea level, respiratory rate, blood pressure and age $>65$ years) on ED admission. Patients were considered HIV-positive for the purpose of this study, either due to previously known chronic infection or as a result of a positive screen conducted in the course of admission. The primary outcome of interest was in-hospital mortality. Secondary outcomes were bacteraemia, ICU admission, orotracheal (OT) intubation and length of hospital stay.

The study was granted ethical approval by the Centre's Institutional Review Board.

\section{Statistical analysis}

The dataset was constructed using Excel (Microsoft Systems, Redmond, Washington, USA) and analysed using Stata 13.1 (Stata Statistical Software: Release 13. College Station, Texas, USA: StataCorp LP). A Chi square test and t-test were used to evaluate 
differences in baseline characteristics. Mantel-Haenszel stratification was used to adjust for age differences when comparing categorical outcomes in relation to HIV serostatus. Multivariate analysis was carried out using linear and logistic regression, in order to identify independent outcome predictors. A doublesided $p$ value lower than 0.05 was considered significant. Associations are mainly expressed as odds ratios (OR) and adjusted odds ratios (aOR). Confidence intervals $(\mathrm{CI})$ are shown for $95 \%$ levels.

\section{Results}

Over the study period, a total of 29,684 adult patients were admitted, 753 of whom for clinically and radiologically (chest radiograph or CT-scan)-confirmed pneumonia. After exclusion of 242 patients for healthcare-associated pneumonia and a further 199 for immunosuppression due to other causes, a total of 396 patients met the study inclusion criteria: 49 HIVpositive and 347 HIV-negative (Table 1). HIV-positive patients were overall younger, predominantly men, with a higher proportion of smokers and lower prevalence of diabetes and heart failure.

Data were available for calculation of CURB-65 in $91.4 \%(n=362)$ of all patients. Mean CURB-65 scores were 1.3 points in HIV-positive and 2.2 points in HIV-negative patients $(p<0.0001$; age adjusted $p=0.44)$.

In-hospital mortality was $4.1 \%(n=2)$ for HIVpositive and $12.1 \%(n=42)$ for HIV-negative patients (age aOR 1.1 CI [0.2-6.3] $p=0.90$ ). Bacteraemia was documented in $8.9 \%(n=35)$ of all patients. Blood cultures drawn from HIV-positive patients were more likely to be positive (aOR 9.1 CI [3.1-27.2] $p<0.001$ ). In all, $11.6 \%(n=46)$ were eventually admitted to an ICU and $9.6 \%(n=38)$ orotracheally intubated in the course of their admission. HIV-positivity did not result in significant differences either in ICU admission (aOR
1.9 CI [0.7-4.8], $p=0.20$ ) or OT intubation (aOR 1.4 CI $[0.5-4.4] p=0.58)$. Mean in-patient stay was 11.6 days, 9.3 for HIV-positive and 12.0 days for HIVnegative patients $(p=0.12$; age-adjusted $p=0.50)$. Stratifying CAP severity according to CURB-65 scores into low, intermediate and high risk, ${ }^{3,25}$ there was a significant trend for higher mortality both in HIV-positive and HIV-negative patients, whereas associations with ICU admission and OT intubation were significant only in either one of the serogroups (Table 2). Receiver operating characteristics (ROC) curves and their respective areas under the curves (AUCs) are depicted in Figure 1.

Lymphocyte subset determinations were available in $96 \%(n=47)$, viral load and HAART use in $98 \%$ $(n=48)$ of all HIV-positive patients. The median CD4+T-lymphocyte cell count was 278 cells $/ \mu \mathrm{L}$ (inter-quartile range 111-541). A majority (58\%, $n=28)$ was on HAART, $40 \%(n=20)$ having achieved viral load suppression in the latest determination. Linear regression showed a negative correlation between CD4 count and length of stay (Figure 2).

Adjusting for comorbidities and CURB-65 score values on multivariate logistic regression, HIVseropositivity did not independently predict mortality (aOR 1.1 CI $[0.2-5.4] p=0.93$ ), OT intubation (aOR $2.9[0.9-9.2] p=0.08$ ) or ICU admission (aOR $1.9 \mathrm{CI}$ [0.7-5.4] $p=0.23$ ). It did however predict bacteraemia (aOR 7.1 CI [2.6-19.5] $p<0.001$ ). Subgroup analyses within HIV-positive patients depicted in Table 3, using HIV-negative patients as a comparator, showed that patients with CD4 counts below 200 cells $/ \mu \mathrm{L}$ were at higher risk of ICU admission and OT intubation, whereas patients with counts above that value were not. No independent effect was seen for different subgroups of viral load suppression and HAART use regarding these outcomes. No subgroup was associated with different mortality and all were associated with higher blood culture positivity.

Table I. Baseline characteristics according to patient serostatus.

\begin{tabular}{lccc}
\hline Baseline variables $(n=396)$ & HIV-positive $(n=49)$ & HIV-negative $(n=347)$ & $p$ Value \\
\hline Men & $38(77.6 \%)$ & $191(55.0 \%)$ & 0.003 \\
Age (mean \pm SD) (years) & $45.3 \pm 13.5$ & $67.8 \pm 21.2$ & $<0.000$ I \\
Smoking & $21(42.9 \%)$ & $77(22.2 \%)$ & 0.001 \\
Diabetes & $1(2.0 \%)$ & $74(21.3 \%)$ & 0.001 \\
Heart failure & $3(6.1 \%)$ & $96(27.7 \%)$ & 0.001 \\
COPD & $9(18.4 \%)$ & $95(27.4 \%)$ & 0.2 \\
CKD & $2(4.1 \%)$ & $31(8.9 \%)$ & 0.4 \\
\hline
\end{tabular}

SD: standard deviation; COPD: chronic obstructive lung disease; CKD: chronic kidney disease. 
Table 2. Risk stratification according to CURB-65 and its association with outcomes.

\begin{tabular}{|c|c|c|c|c|c|}
\hline & \multirow[b]{2}{*}{ CURB-65 mortality risk score } & \multirow[b]{2}{*}{ n (\%) } & \multicolumn{3}{|c|}{ Chi square test for trend } \\
\hline & & & Mortality & OT intubation & ICU admission \\
\hline \multirow{3}{*}{ HIV-negative $(n=3 \mid 7)$} & Low $(0-1)$ & 99 (3I.2\%) & $p<0.001$ & $p=0.04$ & $p=0.17$ \\
\hline & Intermediate (2) & $85(26.8 \%)$ & & & \\
\hline & High (3-5) & I 33 (43.0\%) & & & \\
\hline \multirow[t]{3}{*}{ HIV-positive $(n=48)$} & Low $(0-1)$ & $28(58.3 \%)$ & $p=0.03$ & $p=0.07$ & $p=0.003$ \\
\hline & Intermediate (2) & $16(33.3 \%)$ & & & \\
\hline & High (3-5) & $4(8.3 \%)$ & & & \\
\hline
\end{tabular}

OT: orotracheal; ICU: intensive care unit.

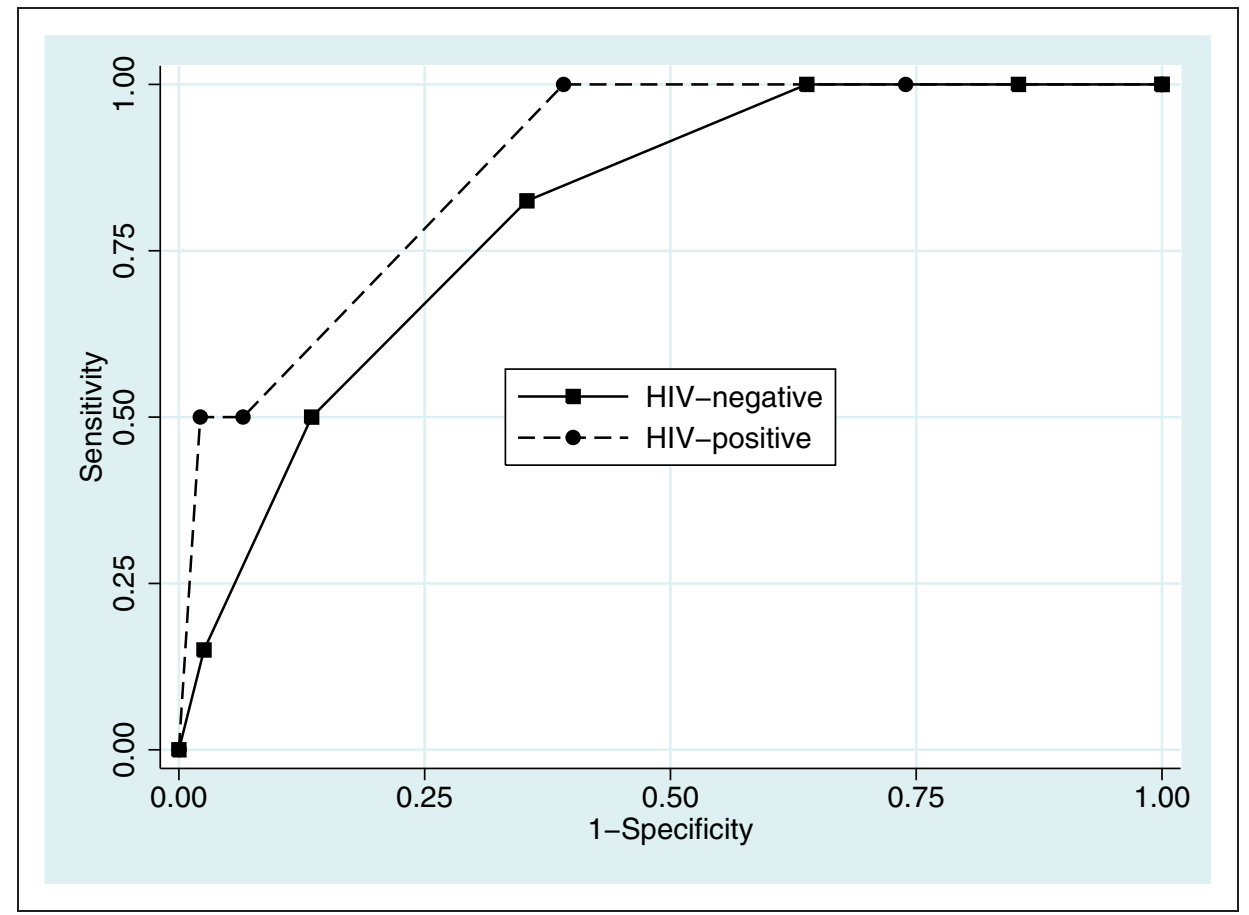

Figure I. ROC curves illustrating performance of CURB-65 in predicting mortality for HIV-positive (AUC 0.880) and HIV-negative patients (AUC 0.804).

\section{Discussion}

Our study suggests that CURB-65 scores on ED presentation are useful in discriminating mortality risk groups, regardless of HIV serostatus. HIV infection overall was associated with lower CURB-65 scores, higher rates of bacteraemia and similar CURB-65controlled clinical outcomes. However, the subgroup of HIV-positive patients with CD4 counts lower than 200 cells $/ \mu \mathrm{L}$ had a higher chance of being admitted to an ICU and of being intubated.

To our knowledge, this is the first work designed to study the CURB-65 score among HIV-positive patients and its findings suggest this score's prognostic performance is maintained in this population. Its reduced number of easily measurable variables makes its use very convenient in busy EDs and primary care centres. Compared to other scores such as PSI, its performance as a prediction rule has been widely validated, ${ }^{26,27}$ one large meta-analysis indicating it might be less sensitive but more specific. $^{26}$

HIV-positive patients in this study had significantly lower severity scores than their seronegative counterparts, similar to what was observed in the CAPO international cohort study. ${ }^{20,21}$ In our case, the difference can be explained by HIV-positive patients being younger, as age $>65$ years is a prognostic feature which contributes one point in the CURB-65 score, and there were no differences with respect to agecontrolled scores. This finding means other unaccounted 


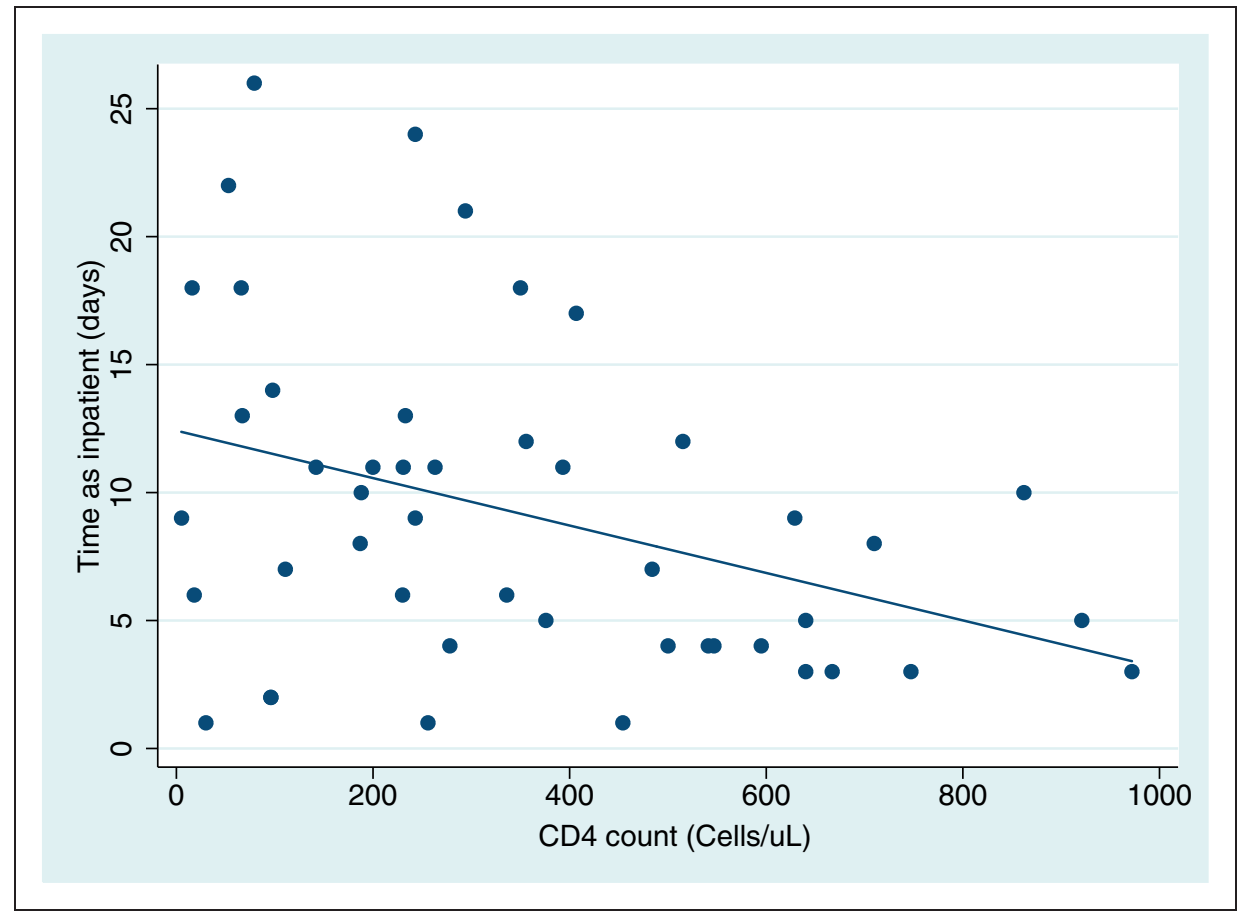

Figure 2. Length of hospital stay as a function of CD4 cell count in HIV-positive patients (linear regression slope $-0.0 \mathrm{I} \mathrm{Cl}$ $[-0.02 ;-0.002] p=0.01)$.

factors guided clinicians in their decision to admit. One possible explanation could be that, compared to the overall population, HIV-positive patients have a higher prevalence of drug use and/or social vulnerability, making more individuals among them unreliable for outpatient therapy. Another potential reason could be that clinicians perceived HIV infection itself as a risk factor lowering the threshold for admission, as was proposed by Malinis et al. ${ }^{21}$ regarding the CAPO study. Both factors could have confounded our study.

The fact that there is a linear relation between CD4 cell count and length of stay and that a stratum-specific independent effect was observed for patients with CD4 cell counts $<200$ cells $/ \mu \mathrm{L}$ regarding ICU admission and OT intubation suggests that this variable played a role in all these outcomes. Admission periods could have been protracted by a longer time to achieve clinical stability, but the higher risk of concomitant opportunistic diseases is also a consideration. More frequent ICU admission and OT intubation leads us to hypothesise that a greater impairment of cellular immunity put patients at risk of respiratory failure and/or severe sepsis. Accordingly, research has shown that CD4 cell depletion not only contributes to bloodstream invasion by Streptococcus pneumoniae but also to deregulated inflammatory response through impaired release of proinflammatory cytokines. $^{28}$

Stemming from different methodologies, there is disagreement in the association between lower CD4 cell counts and worse outcomes, including multilobar consolidation, longer time to clinical stability and increased mortality. Some studies have reported no impact of this marker on CAP outcome, ${ }^{11,17}$ whereas others have conversely found a significant impact on mortality, prompting authors to recommend admission of all patients in the $<200$ cells $/ \mu \mathrm{L}$ subgroup. $^{13,15,29}$ Taking into account our results, in addition to CURB-65 scores, low CD4 cell counts seem to be independent predictive factors in the severity assessment of HIV-positive patients presenting with CAP. Both may thus be taken into consideration to aid clinical judgment when deciding site-of-care.

The major limitation of this study was the relatively small size of its HIV-positive cohort and the limited number of primary events in this population. Other limitations include its retrospective design and the fact that it was conducted in a single urban tertiary centre, which may limit its generalisability. Nevertheless, it is expected that our assumptions apply to other so-called high-income settings where HAART is widely accessible. Not all patients with unknown serostatus were tested for HIV, which could have resulted in underestimation of seropositivity and misclassification bias. Furthermore, patients presenting with CAP who were discharged from the ED were not included, a constraint of virtually all major works on this topic. 
Table 3. CURB-65 controlled, comorbidity-adjusted outcomes among specific sub-groups of HIV-infected individuals according to CD4 count, viral load and HAART use $(n=49)$.

\begin{tabular}{|c|c|c|c|c|}
\hline $\begin{array}{l}\text { HIV positive patient } \\
\text { sub-group }\end{array}$ & Mortality & OT intubation & ICU admission & Bacteraemia \\
\hline$>200 \mathrm{CD} 4$ cells $/ \mu \mathrm{L}$ & $\begin{array}{l}\text { OR } 0.8 \\
\mathrm{Cl}[0.1-6.6] \\
p=0.80\end{array}$ & $\begin{array}{l}\text { OR } 0.8 \\
\mathrm{Cl}[0.1-6.5] \\
p=0.83\end{array}$ & $\begin{array}{l}\text { OR } 2.2 \\
\mathrm{Cl}[0.7-7.6] \\
p=0.20\end{array}$ & $\begin{array}{l}\text { OR 7.I } \\
\text { CI }[2.5-20.2] \\
P<0.00 I\end{array}$ \\
\hline$<200$ CD4 cells $/ \mu \mathrm{L}$ & $\begin{array}{l}\text { OR I.7 } \\
\mathrm{Cl}[0.2-15.2] \\
p=0.64\end{array}$ & $\begin{array}{l}\text { OR 9.I } \\
\text { CI }[2.2-37.1] \\
p=0.002\end{array}$ & $\begin{array}{l}\text { OR } 5.7 \\
\text { CI }[1.5-22.0] \\
p=0.01\end{array}$ & $\begin{array}{l}\text { OR } 6.7 \\
C I[1.8-25.4] \\
p=0.005\end{array}$ \\
\hline$<50$ viral copies $/ \mathrm{mL}$ & $\begin{array}{l}\text { OR I.I } \\
C I[0.1-10.7] \\
p=0.4 I\end{array}$ & $\begin{array}{l}\text { OR } 2.9 \\
C I[0.6-\mid 5.1] \\
p=0.20\end{array}$ & $\begin{array}{l}\text { OR 3.I } \\
\mathrm{Cl}[0.9-10.2] \\
p=0.07\end{array}$ & $\begin{array}{l}\text { OR } 4.2 \\
\text { CI }[1.0-\mid 7.0] \\
p=0.05\end{array}$ \\
\hline$>50$ viral copies/mL & $\begin{array}{l}\text { OR 2.I } \\
\mathrm{Cl}[0.2-2 \mid .2] \\
p=0.53\end{array}$ & $\begin{array}{l}\text { OR2.5 } \\
\mathrm{CI}[0.6-10.4] \\
p=0.21\end{array}$ & $\begin{array}{l}\text { OR } 2.0 \\
\mathrm{Cl}[0.6-7.1] \\
p=0.27\end{array}$ & $\begin{array}{l}\text { OR 9.I } \\
\text { Cl [3.3-25.3] } \\
p<0.001\end{array}$ \\
\hline On HAART & $\begin{array}{l}\text { OR } 0.8 \\
\mathrm{Cl}[0.1-6.6] \\
p=0.80\end{array}$ & $\begin{array}{l}\text { OR 3.I } \\
\mathrm{CI}[0.8-12.3] \\
p=0.11\end{array}$ & $\begin{array}{l}\text { OR } 2.5 \\
\mathrm{Cl}[0.9-7.3] \\
p=0.09\end{array}$ & $\begin{array}{l}\text { OR } 6.3 \\
\text { CI }[2.0-19.3] \\
p=0.001\end{array}$ \\
\hline Not on HAART & $\begin{array}{l}\text { OR I.8 } \\
\text { Cl }[0.2-17.0] \\
p=0.60\end{array}$ & $\begin{array}{l}\text { OR 2.I } \\
\mathrm{Cl}[0.4-\mathrm{II} .8] \\
p=0.38\end{array}$ & $\begin{array}{l}\text { OR } 2.0 \\
\mathrm{Cl}[0.5-8.4] \\
p=0.35\end{array}$ & $\begin{array}{l}\text { OR } 9.0 \\
\text { CI [2.8-28.8] } \\
p<0.00 I\end{array}$ \\
\hline
\end{tabular}

OT: oro-tracheal; ICU: intensive care unit; HAART: highly-active anti-retroviral therapy; OR: odds ratio; Cl: confidence interval. Statistically significant results are presented in bold characters.

A larger multi-centric study is imperative to develop a reliable prediction model, which takes into account specific aspects of HIV patients with CAP.

\section{Conclusions}

The performance of CURB-65 in predicting mortality appears to be maintained in HIV-positive patients. HIV infection overall was not an intrinsic independent severity predictor in patients presenting with CAP. However, patients with lower CD4 cell counts may have a higher risk for OT intubation, ICU admission and longer admission periods. In addition to using severity assessment scores such as CURB-65, clinicians should also bear in mind the prognostic value of CD4 counts.

\section{Declaration of Conflicting Interests}

The authors declared no potential conflicts of interest with respect to the research, authorship, and/or publication of this article.

\section{Funding}

The authors received no financial support for the research, authorship, and/or publication of this article.

\section{Acknowledgements}

We thank Ana Lladó, Pedro E. Silva, Catarina Patrício, Filipa Silva, Joana Ferreira, João Oliveira, Lara Câmara,
Rodrigo Leão, Rui Malheiro, Mário Silva, Pedro Russo, Pedro la Féria and Paulo Barreto for their contribution in data gathering; Daniel Virella and Marta Alves for their help in building the study framework. Finally, a special thanks to Pedro Varela for his help in artwork conception.

\section{References}

1. Palella FJ Jr, Delaney KM, Moorman AC, et al. Declining morbidity and mortality among patients with advanced human immunodeficiency virus infection. $N$ Engl J Med 1998; 338: 853-860.

2. Montaner JS, Lima VP, Harrigan PR, et al. Expansion of HAART coverage is associated with sustained decreases in HIV/AIDS mortality and HIV transmission: the 'HIV Treatment as Prevention' experience in a Canadian setting. Plos One 2014; 12: e87872.

3. Lim WS, Baudouin SV, George RC, et al. British Thoracic Society guidelines for the management of community acquired pneumonia in adults: update 2009. Thorax 2009; 64: iii1-iii55.

4. Froes F, Paiva JA, Amaro P, et al. Consensus document on nosocomial pneumonia. Rev Port Pneumol 2007; 13: $419-486$.

5. Jones N, Huebner R, Khoosal M, et al. The impact of HIV on Streptococcus pneumoniae bacteraemia in a South African population. AIDS 1998; 12: 2177-2184.

6. Kohli R, Lo Y, Homel P, et al. Bacterial pneumonia, HIV therapy, and disease progression among HIV-infected women in the HIV epidemiologic research (HER) study. Clin Infect Dis 2006; 43: 90-98. 
7. Heffernan RT, Barrett NL, Gallagher KM, et al. Declining incidence of invasive Streptococcus pneumoniae infections among persons with AIDS in an era of highly active antiretroviral therapy, 1995-2000. J Infect Dis 2005; 191: 2038-2045.

8. Felkin DR, Feldman C, Schuschat A, et al. Global strategies to prevent bacterial pneumonia in adults with HIV disease. Lancet Infect Dis 2004; 4: 445-55.

9. Center for Diseases Control and Prevention. Revised Surveillance Case Definition for HIV Infection - United States, 2014. Report, CDC, Atlanta, 2014.

10. World Health Organization. WHO Case Definitions of HIV for Surveillance and Revised Clinical Staging and Immunological Classification of HIV-Related Disease in Adults and Children. Report, WHO, Geneva, 2007.

11. Perelló R, Escoda O, Camon S, et al. Changes in the etiology, incidence and prognosis of acute lower respiratory tract infections in human immunodeficiency virus patients. Enferm Infecc Microbiol Clin 2015; 33: 243-247.

12. Søgaard OS, Reekie J, Ristola M, et al. Severe bacterial non-AIDS infections in HIV-positive persons: incidence rates and risk factors. $J$ Infect 2013; 66: 439-446.

13. Curran A, Falcó V, Crespo M, et al. Bacterial pneumonia in HIV-infected patients: use of the pneumonia severity index and impact of current management on incidence, aetiology and outcome. HIV Med 2008; 9: 609-615.

14. Afessa B and Green B. Bacterial pneumonia in hospitalized patients with HIV infection: the pulmonary complications, ICU support, and prognostic factors of hospitalized patients with HIV (PIP) Study. Chest 2000; 117: 1017-1022.

15. Madeddu G, Laura Fiori $\mathbf{M}$ and Stella Mura M. Bacterial community-acquired pneumonia in HIVinfected patients. Curr Opin Pulm Med 2010; 16: 201-207.

16. Madeddu G, Monforte AD, Girardi E, et al. CD4 cell count and the risk of infective and non-infective serious non-AIDS events in HIV-positive persons seen for care in Italy. J Int AIDS Soc 2014; 17: 19509.

17. Chew KW, Yen IH, Li JZ, et al. Predictors of pneumonia severity in HIV-infected adults admitted to an urban public hospital. AIDS Patient Care STDS 2011; 25: 273-277.

18. Sanders KM, Marras TK and Chan CK. Pneumonia severity index in the immunocompromised. Can Respir $J$ 2006; 13: 89-93.
19. Mégarbane B, Bruneel F, Chevret S, et al. Severe community-acquired pneumonia from Streptococcus pneumoniae in HIV-infected patients: epidemiology and prognostic features of mortality. Pathol Biol 1999; 47: 422-429.

20. Christensen D, Feldman C, Rossi P, et al. HIV infection does not influence clinical outcomes in hospitalized patients with bacterial community-acquired pneumonia: results from the CAPO international cohort study. Clin Infect Dis 2005; 41: 554-556.

21. Malinis M, Myers J, Bordon J, et al. Clinical outcomes of HIV-infected patients hospitalized with community acquired pneumonia. Int J Infect Dis 2010; 14: e22-27.

22. Mandell LA, Wunderink RG, Anzueto A, et al. Infectious Diseases Society of America/American Thoracic Society consensus guidelines on the management of community-acquired pneumonia in adults. Clin Inf Dis 2007; 44 Suppl 2: S27-72.

23. Fine MJ, Auble TE, Yealy DM, et al. A prediction rule to identify low-risk patients with community-acquired pneumonia. N Engl J Med 1997; 336: 243-250.

24. Albrich WC, Madhi SA, Adrian PV, et al. Pneumococcal colonization density: a new marker for disease severity in HIV-infected patients with pneumonia. BMJ Open 2014; 48: e005953.

25. Mbata GC, Chukwuka CJ, Onyedum CC, et al. The CURB-65 scoring system in severity assessment of Eastern Nigerian patients with community-acquired pneumonia: a prospective observational study. Prim Care Respir J 2013; 22: 175-180.

26. Loke YK, Kwok CS, Niruban A, et al. Value of severity scales in predicting mortality from community-acquired pneumonia: systematic review and meta-analysis. Thorax 2010; 65: 884-890.

27. Varshochi M, Kianmehr P, Naghavi-Behzad M, et al. Correspondence between hospital admission and the pneumonia severity índex (PSI), CURB-65 criteria and comparison of their predictive value in mortality and hospital stay. Infez Med 2013; 21: 103-110.

28. Huson M, Grobusch A and van der Poll T. The effect of HIV infection on the host response to bacterial sepsis. Lancet Infect Dis 2015; 15: 95-108.

29. Hoffmann C and Rockstroh JK. Bacterial pneumonia. In: Hoffmann C (ed.) HIV 2012/2013. Hamburg: Medizin Fokus Verlag, 2012, pp.387-389. 